\title{
A study on the antibiotic susceptibility of Staphylococcus aureus from nasal samples of female students at the Obafemi Awolowo university campus
}

\begin{abstract}
One of the predominant bacteria found in the nose is Staphylococcus aureus, and they are found in approximately the same number as on the skin and face. This study was carried out to isolate and analyze the antibiotic susceptibility of Staphylococcus aureus gotten from the nasal cavity of female students (at undergraduate and postgraduate levels) of Obafemi Awolowo University Ile-Ife campus, Osun state, Nigeria. Out of 40 samples obtained from the nasal cavity of the female students, a total of 9 isolates of Staphylococcus aureus were confirmed. Based on the biochemical tests, they were all Gram-positive cocci in clusters, catalase positive, coagulase positive and DNase positive. The antibiotic susceptibility testing showed that $88.9 \%$ were resistant to penicillin. Therefore, to avoid infection by drug resistant Staphylococcus aureus, people must ensure that their nose is kept clean and should practice good hygiene especially in hospital environments to prevent nosocomial infections due to Staphylococcus aureus.
\end{abstract}

Keywords: Staphylococcus aureus, Antibiotic susceptibility test, Gram-positive cocci, DNase catalase, coagulase, catalase
Volume 7 Issue 2 - 2019

\author{
Taiwo Mary Makinde,' Kwashie Ajibade Ako- \\ $\mathrm{Nai}^{2}$ \\ 'Department of Biological Sciences, National University of Life \\ and Environmental Sciences of Ukraine, Ukraine \\ ${ }^{2}$ Department of Microbiology, Faculty of Science, Obafemi \\ Awolowo University, Nigeria
}

\author{
Correspondence: Taiwo Mary Makinde, Department \\ of Biological Sciences, National University of Life and \\ Environmental Sciences of Ukraine, Heroiv Oborony St, 15, Kyiv, \\ 0304I, Ukraine, Tel +380 733062230 \\ Email Lovebiotechnology123@gmail.com
}

Received: February 27, 2019 | Published: March 25, 2019

\section{Introduction}

The visible part of the human nose is the protruding part of the face that bears the nostrils. The shape of the nose is determined by the ethmoid bone and the nasal septum, which consists mostly of cartilage and which separates the nostrils. On the average the nose of a female is smaller than that of a male. The nose has an area of specialized cells which are responsible for smelling (part of the olfactory system). Another function of the nose is the conditioning of the inhaled air, warming it and making it more humid. Hairs inside the nose prevent large particles from entering the lungs. Sneezing is usually caused by foreign particles irritating the nasal mucosa but can more rarely be caused by sudden exposure to bright light (called the photic sneeze reflex) or touching the external auditory canal. Sneezing is a means of transmitting infections because it creates aerosols in which the droplets can harbor microbes. ${ }^{1}$

The nasal root is the top of the nose, forming an indentation at the suture where the nasal bone meets the frontal bone. The anterior nasal spine is the thin projection of the bone at the midline on the lower nasal margin, holding the cartilaginous center of the nose. The mammalian respiratory system has formidable defense mechanism. The average person inhales at least eight microorganisms a minute or 10,000 each day. Once inhaled, a microorganism must first survive and penetrate the air filtration system of the upper and lower respiratory tracts. Because the airflow in this tract is turbulent, microorganisms are deposited on the moist, sticky mucosal surface. The cilia in the nasal cavity beat towards the pharynx so that the mucus with its trapped microbes is moved toward the mouth and expelled. ${ }^{2}$ The normal microbiota of the nose is found just inside the nostrils. $S$. aureus and $S$. epidermis are the predominant bacteria present and are found in approximately the same numbers as on the skin and face. ${ }^{2}$ About $20 \%$ of healthy people almost never carry $S$. aureus, about $60 \%$ have $S$. aureus from time to time and about $20 \%$ almost never carry $S$. aureus. ${ }^{1}$

\section{Staphylococci}

Staphylococcus is a common bacterium found on the skin and in the nose of the healthy people. The same comes from a Greek word staphyle, which means a bunch of grapes and kokkos which means berry. Staphylococci look like a bunch of grapes or berries, when observed under a microscope. These gram-positive bacteria are facultative anaerobic organisms. These bacteria normally do not cause infectious diseases when living on the skin or in the nose. Staphylococci are opportunistic pathogens that cause skin disorders when they gain entry in the body through cuts, wounds or damage to the skin. There are many Staphylococcal infections that affect the nose. Impetigo, furuncles and carbuncles are common staphylococcal infections of the nose. Taxonomically, the genus Staphylococcus is in the Bacterial family Staphylococcaceae, which includes three lesser known genera, Gamella, Macrococcus and Salinicoccus. The best known of its nearby phylogenetic relatives are the members of the genus Bacillus in the family Bacillaceae, which is on the same level as the family Staphylococcaceae. The Listeriaceae are also a nearby family.

Staphylococci are facultative anaerobes that grow by anaerobic respiration or by fermentation that yields principally lactic acid. $S$. aureus can grow at a temperature range of $15^{\circ} \mathrm{C}-45^{\circ} \mathrm{C}$ and at $\mathrm{NaCl}$ concentration as high as $15 \% .^{1}$ Staphylococci are non-motile, non-spore forming and catalase positive. Their cell wall contains peptidoglycan and teichoic acid. ${ }^{4}$ Staphylococcus aureus often produce golden yellow colonies, often with hemolysis when grown on blood agar plates. ${ }^{5}$ More than $90 \%$ of $S$. aureus are resistant to the beta-lactamase- resistant penicillin such as methicillin, Nafcillin and Oxacillin, by virtue of changes in the penicillin-binding proteins in their cell membranes. These strains are commonly known as Methicillin resistant $S$. aureus (MRSA) (Figure 1).

\section{The important phenotypic characteristics of} Staphylococcus aureus are:

a. Gram-positive, cluster-forming coccus

b. Non-motile, non-spore forming facultative anaerobe

c. Fermentation of glucose produces mainly lactic acid

d. Ferments mannitol (distinguishes from S. epidermis) 
e. Catalase positive and coagulase positive

f. Golden yellow colony on agar

g. Normal flora of human found on nasal passages, skin and mucous membranes

h. Pathogen of humans causes a wide range of suppurative infections, as well as food poisoning and toxic shock syndrome.

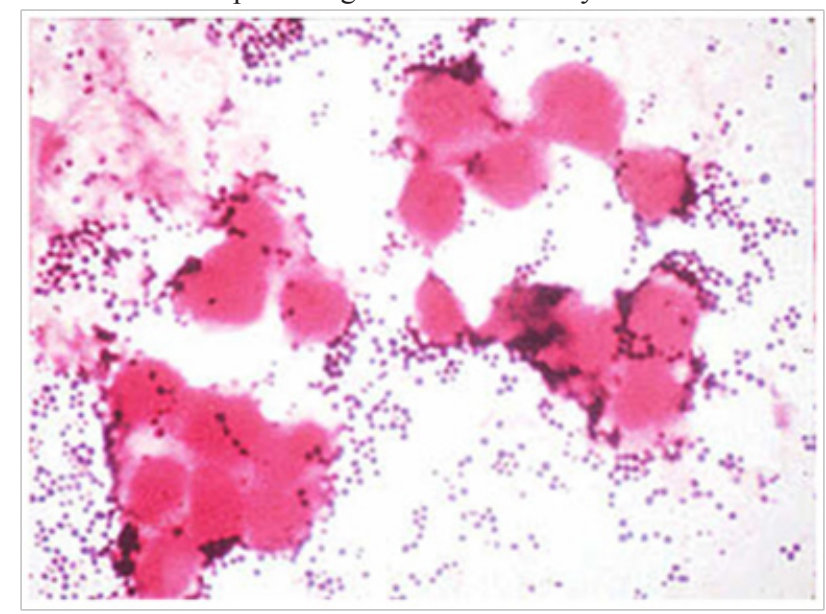

Figure I Gram stain of Staphylococcus aureus in pustular exudates.

Penicillin's are classified as $\beta$-lactam drugs because of their unique four-member lactam ring. They share features of chemistry, mechanism of action, pharmacological and clinical effects, and immunologic characteristics with cephalosporins, monobactams, carbapenems and $\beta$-lactam compounds. ${ }^{7}$ All penicillin's have the basic structure of a thiazolidine ring attached to a $\beta$-lactam ring that carries a secondary amino group (RNH). Substituents (R) can be attached to the amino group. Structural integrity of the 6-amino penicillin acid nucleus is essential for the biologic activity of these compounds. If the $\beta$-lactam ring is enzymatically cleaved by $\beta$-lactamases, the resulting product, penicilloic acid, lacks antibacterial activity. ${ }^{8}$ The aim of this study is to isolate and analyze Staphylococcus aureus gotten from the nasal cavity of female students (at undergraduate and postgraduate levels) of Obafemi Awolowo University Ile-Ife campus, Osun state, Nigeria. The study population consisted of forty randomly selected healthy female students. It was conducted between August and October 2010

\section{Antimicrobial resistance}

Drug resistance is an extremely serious public health problem. Much of the difficulty arises from drug misuse. Most times, antibiotics are prescribed without culturing or identifying the pathogen or without determining bacteria sensitivity to the drug. Broad spectrum drugs are sometimes given in place of narrow spectrum drugs as a substitute for culture and sensitivity with the consequent risk of dangerous side effects, super infections and the selection of drug resistant mutants. ${ }^{2}$ Penicillin $\mathrm{G}$ was the first antibiotic to be used in medicine. It has a $\beta$ - lactam ring which is attached to a 5 -member ring which is active against staphylococci. Cephalosporins (cephalexin, cefuroxime, cefotaxime) are all active against gram positive cocci. ${ }^{9}$

\section{Mechanism of action}

Penicillin like all $\beta$-lactam antibiotics, inhibit bacterial growth by interfering with a Specific step in bacteria cell walls synthesis. The cell wall is a rigid outer layer that is not found in animal cells. It surrounds the cytoplasmic membrane, maintaining the shape of the cell and preventing cell lysis from high osmotic pressure. ${ }^{10}$ The cell wall is composed of complex cross-linked polymer, peptidoglycan/murein consisting of polysaccharides and polypeptides. The polysaccharide contains alternating amino sugars, $\mathrm{N}$-acetylglucosamine and $\mathrm{N}$-acetylmuramic acid. A five -amino acid peptide is linked to the $\mathrm{N}$ acetylmuramic acid sugar. This peptide terminates in the D-alanyl-Dalanine penicillin binding proteins (PBPs) catalyze the transpeptidase reaction that removes the terminal alanine to form a Crosslink with a nearby peptide which gives rigidity. B ell walls its structural lactam antibiotics are structural analogs of the la-D-Ala substrate and they are covalently B -lactam antibiotic has attached to the PBP, the bound by PBPs at the active site. After a is synthesis is blocked and responsible for cell transpeptidation reaction inhibited, peptidoglycan death, Penicillin's and cephalosporins are bactericidal only if cells are actively growing and synthesizing cell wall. ${ }^{9}$

\section{Classification}

The attachment of different substituents to the G- amino penicillin acid terminus the essential pharmacological and antibacterial properties of the resulting molecules; penicillin can be assigned to one of three groups. Within each of the groups are compounds that are relatively stable to gastric acid and suitable for oral administration, e.g. Penicillin V, Dicloxacillin and Amoxicillin.

a. Penicillin (e.g. penicillin G): These have the greatest activity against Gram positive organisms, gram negative cocci and non $\beta$-lactamase producing anaerobes, however they have little activity against Gram- rods. They are susceptible to hydrolysis by $\beta$-lactamase.

b. Anti-staphylococcal penicillin's: Examples of this penicillin include methicillin, nafcillin, isoxazolyl, penicillin's like Oxacillin, cloxacillin or dicloxacillin and flucloxacillin. They are active against staphylococci and streptococci but inactive against enterococci, anaerobic organisms and gram-negative cocci and rods.

c. Extended- spectrum penicillin's: These drugs retain the antibacterial spectrum of penicillin's and have improved activity against gram negative organisms but are destroyed lactamase ${ }^{11}$ they by B- are subdivided into 3 . They are:

i. Aminopenicillins (Ampicillin, Amoxycillin): they have the same spectrum and activity. But amoxicillin is the most active of the oral B- lactam antibiotics against penicillin-resistant pneumococci. ${ }^{12}$

ii. Carboxypenicillins (carbenicillin, ticarcillin) carbenicillin, the first antipseudomonal carboxypenicillin. It is acid stable and can be given orally in urinary tract infection. Ticarcillin is like carbenicillin in activity but effective in lower doses.

iii. Ureidopenicillins (piperacillin, mezlocillin, azlocillin). They resemble Ticarcillin except that they are also active against selective gram-negative bacilli, such as klebsiella. Ampicillin, Amoxillin, ticarcillin and Piperacillin are also available in combination with one of several B- lactamase inhibitor that extends the activity of these penicillin's to include $\beta$-lactamaseproducing strains of $\mathrm{S}$. aureus as well as some B-lactamaseproducing gram: negative bacteria. 


\section{Resistance to penicillins}

Resistance to Penicillin and other $\beta$-lactam is due to some general mechanisms. They are:

a) Inactivation of antibiotic by B- lactamase production. More than 50 different $\beta$-lactamases are known, most of them produced under the control of bacterial plasmids. Some $\beta$-lactamases are inducible by the newer cephalosporins. ${ }^{9}$

b) Modification of target PBPs.

c) Impaired penetration of drug to target PBPs. Resistance caused by impaired penetration of antibiotic to target PBPs, which occurs only in gram- -negative species, is due to impermeability of an outer cell membrane that is present in Gram- negative but not in Gram-positive organisms. B-Lactam antibiotics cross the outer membrane and enter the Gram- negative organisms via the outer membrane protein channels, (porins). Absence of proper channel or down regulation of its production can prevent or greatly reduce drug entry into the cell. ${ }^{7}$

d) The presence of an efflux pump. Gram-negative organism also may produce an efflux pump, which consists of cytoplasmic and periplasmic protein component that efficiently transport some $\beta$-lactam antibiotics back across the outer membrane.

e) Failure to synthesize peptidoglycans e.g. in mycoplasma, L forms are metabolically inactive bacteria. ${ }^{9}$

\section{Treatment and infection control}

Penicillin can be used to treat S aureus infections; but, in most countries, penicillin resistance is extremely common and first-line therapy is most commonly a penicillinase-resistant $\beta$-lactam antibiotic (for example, oxacillin or flucloxacillin). Combination therapy with gentamicin may be used to treat serious infections like endocarditis, but its use is controversial because of the high risk of damage to the kidneys. The duration of treatment depends on the site of infection and on severity. ${ }^{13}$ Emphases on basic hand washing techniques are effective in preventing the transmission of S. aureus. The use of disposable aprons and gloves by staffs in the hospitals reduces the risk of transmission. Staff or patients who are found to carry resistant strains of S. aureus may be required to undergo eradication therapy which may include antiseptic washes and shampoos such as chlorhexidine and application of topical antibiotic ointments (such as mupirocin or neomycin) to the anterior nares of the nose. ${ }^{14}$

Application of Vaseline inside the nose when suffering from cuts in the nose is advisable; it should be applied twice a day for 10 days. The standard treatment for staphylococcal infection in nose is done by use of antibiotic ointments. These over-the-counter ointments can help get rid of the Staphylococcal infections in nose. Many times, these infections do not subside for over a Year Doctors may suggest use of medicinal honey that contains antibacterial properties that may inhibit further growth of staphylococci. Abscesses can also be drained surgically. The doctor may decide on an antibiotic staph infection treatment after referring to the culture results in the laboratory. The highly resistant strains or serious infections are treated with intravenous antibiotics. Ethanol has proven to be an effective topical sanitizer against MRSA. Quaternary ammonium can be used in conjunction with ethanol to increase the duration of the sanitizing action. The prevention of nosocomial infections involves routine and terminal cleaning. Nonflammable alcohol vapor in $\mathrm{CO}_{2} \mathrm{NAV}-\mathrm{CO}_{2}$ systems have an advantage as they do not attack metals or plastics used in medical environments, and do not contribute to antibacterial resistance.

\section{Aims and objectives of this study}

The objective of this study is to determine the effectiveness of penicillin against isolated $S$. aureus obtained from the nasal cavity of female students.

\section{List of materials used}

i. Glass wares: The glass wares used are the conical flasks, cover slips, beakers, and disposable petri-dishes, glass rods, glass slides, measuring cylinder, and pipette, test tubes, and glass test tubes.

ii. Equipment's: Autoclave, Bunsen burner, light microscope, thermostat, centrifuge, cell colony counter, hot plate, hot air oven, weighing balance.

iii. Reagents: Alcohol (95\%), crystal violet, distilled water, gram's iodine, human plasma, hydrogen peroxide, immersion oil and safranin.

iv. Media: Mannitol salt agar (MSA), DNase Agar, Nutrient broth, normal saline, Mueller Hinton Agar, peptone water and antibiotics.

v. Other materials: Marker, inoculating needle, inoculating loop, sterile swab sticks, paper tape, Aluminum foil, cotton wool, vials, test tube rack, forceps, disinfectant, Antibiotic disc.

\section{Methods}

\section{Sterilization of materials}

This is a process whereby microorganisms are inactivated, killed or removed from a material. This is done to avoid contamination. It involves the use of an autoclave which uses steam under pressure. Materials are sterilized in the autoclave at $121^{\circ} \mathrm{C}$ for 15 minutes.

\section{Collection of samples}

Nasal swab samples were obtained by using sterile swab sticks which has been moistened with physiological normal saline. Both the left and the right anterior nares were swabbed by rubbing the swab stick around the inside of each nostril while applying an even pressure and rotating the swab without interruption. The swab was then placed back into its container and sealed. The swabs were then labeled and taken to the laboratory immediately for bacteriological examination.

\section{Process}

\section{Sterilization of other materials}

Materials such as forceps, plastic containers were sterilized using $95^{\circ} \mathrm{C}$ ethanol. Hand and the environment where the work was carried out were also disinfected using $95 \%$ ethanol.

\section{Preparation and sterilization of media}

The media used for this study were Mannitol Salt agar, Mueller Hinton agar, Nutrient Broth and Nutrient Agar. All the media were prepared according to the manufacturer's specifications. The media was weighed and were poured into a measuring cylinder containing distilled water of required volume after which the medium was heated on hot plate to dissolve properly and sterilized by autoclaving at $121^{\circ} \mathrm{C}$ for 15 minutes. 


\section{Composition and preparation of media used in this study}

i. Normal saline: This medium was used for the collection and transportation of specimens from the source into the medium used to culture.

\section{Composition: $0.85 \mathrm{~g} \mathrm{NaCl}$ in $100 \mathrm{ml}$ water.}

Preparation: $0.85 \mathrm{~g}$ of $\mathrm{NaCl}$ powder was weighed into $100 \mathrm{ml}$ of distilled water, shaken to dissolve, then poured into test tubes and then sterilized at $121^{\circ} \mathrm{C}$ for 15 minutes in an autoclave. Then it was left to cool down.

ii. Nutrient broth: This medium was used for culturing bacteria and more fastidious organisms.

Composition: D- Glucose-1.0g/l, Yeast extract-2.0g/1, Peptone$5.0 \mathrm{~g} / \mathrm{l}, \mathrm{NaCl}-5.0 \mathrm{~g} / \mathrm{l}$, Final $\mathrm{pH}=7.5 \pm 0.2$ at $37^{\circ} \mathrm{C}$

Preparation: $13 \mathrm{~g}$ of nutrient broth powder was weighed into 1 liter of distilled water. It was swirled to dissolve, poured into test tubes and sterilized at $121^{\circ} \mathrm{C}$ for 15 minutes.

iii. Manitol salt agar: This medium was used for the selective isolation of Staphylococci.

Composition: Peptic digest of animal tissue- $5 \mathrm{~g} / 1$, Pancreatic digest of casein-5g/1, Beef extract-1g/1, D-Mannitol-10g/l, Sodium Chloride-75g/l, Phenol red-0.025g/1, Agar-15g/1, Final pH=7.4 \pm 0.2 at $37^{\circ} \mathrm{C}$

Preparation: $108 \mathrm{~g}$ of MSA powder was weighed into 1 liter of distilled water, heated to dissolve and then sterilized at $121^{\circ} \mathrm{C}$ for 15 minutes. It was left to cool at $45^{\circ} \mathrm{C}$, poured into Petri dishes and allowed to set.

iv. Nutrient agar: This medium was used for the cultivation and the further study of bacteria.

Composition: Meat extract-1g/1, Yeast extract-2g/1, Peptone-5g/1, Sodium chloride- $5 \mathrm{~g} / \mathrm{l}$, Agar- $15 \mathrm{~g} / \mathrm{l}$, Final $\mathrm{pH}=7.4 \pm 0.2$ at $37^{\circ} \mathrm{C}$

Preparation: $28 \mathrm{~g}$ of nutrient agar was weighed into 1 liter of distilled water, heated to dissolve, sterilized at $121^{\circ} \mathrm{C}$ for 15 minutes. It was then allowed to cool to $45^{\circ} \mathrm{C}$, poured into petri dishes and allowed to set.

v. Mueller hinton agar (MHA):This medium was used for antibiotic sensitivity testing.

Composition: Beef infusion solids- $4 \mathrm{~g} / 1$, Starch- $1.5 \mathrm{~g} / 1$, Casein hydrolysates-17.5g/1, Agar- $15 \mathrm{~g} / 1$, Final $\mathrm{pH}=7.4 \pm 0.2^{\circ} \mathrm{C}$

Preparation: $38 \mathrm{~g}$ of Mueller Hinton Agar (MHA) powder was weighed into 1 liter of distilled water, heated to dissolve, sterilized at $121^{\circ} \mathrm{C}$ for 15 minutes, poured into petri-dishes and allowed to set.

\section{Isolation or organisms}

Each sample obtained on moistened sterile swab sticks from the anterior nares was suspended in $5 \mathrm{ml}$ nutrient broth in a test tube. The culture was then incubated at $37^{\circ} \mathrm{C}$ for 24 hours. Thereafter, the inoculum was streaked on MSA (Mannitol Salt Agar) plates and incubated at $37^{\circ} \mathrm{C}$ for 48 hours. A discrete, yellow, convex, raised colony was picked from the MSA plate using a sterile inoculating needle and sub cultured into nutrient broth and then on nutrient agar for further identification.

\section{Identification tests}

Gram stain: This test is used to distinguish between a Gram -positive and a Gram- negative organism. About 2-3 discrete colonies of the culture were picked using a sterile inoculating loop and a thin smear was prepared on a clean slide. It was heat fixed by passing though the flame of the burner. A drop of crystal violet was added to the smear for 1 minute and washed off with water. It was then flooded with Gram's iodine (a mordant) for 1 minute and washed with water. The stain was decolorized with $95 \%$ alcohol. The secondary stain (carbon fuchsin) was added for 30 seconds and washed with water. The stain was washed and dried and observed under oil objective after the addition of oil immersion. Gram positive organisms stained purple because they retained the purple color of the crystal violetiodine complex. Gram negative organisms stained pink as they were decolorized by the alcohol treatment and therefore retained the color of the carbon fuchsin which served as the counter stain.

Catalase test: This is used to differentiate staphylococci from streptococci which are both gram positive. ${ }^{2}$ A drop of $3 \%$ hydrogen peroxide was placed on a clean glass slide and a colony of the pure isolate from the culture sample was added to it. Staphylococci showed effervescence immediately by converting hydrogen peroxide to oxygen and water.

$$
2 \mathrm{H}_{2} \mathrm{O}_{2} \text { catalase } \rightarrow 2 \mathrm{H}_{2} \mathrm{O}+\mathrm{O}_{2} \uparrow
$$

Coagulase test: It detects the presence of coagulase. Coagulase causes plasma to clot. It is an important test used to differentiate between $S$. aureus (+) from $S$. epidermis (-). ${ }^{2} 0.5 \mathrm{ml}$ of human plasma was added to a test tube containing $0.5 \mathrm{ml}$ of an 18-24 hr. old broth culture of the test culture. The tube was incubated at $37^{\circ} \mathrm{C}$ for 24 hours and examined after 4hours and 24 hours. The presence of clot or coagulation in the tube showed a positive coagulase result, while the absence of clot or coagulation indicated a negative result.

DNase test: The DNase agar is prepared, sterilized and poured aseptically into petri dishes and allowed to dry and set. A sterile inoculating loop is used to streak fresh culture from the nutrient agar on the DNase agar which is incubated at $37^{\circ} \mathrm{C}$ for 24 hours. After incubation, $1 \mathrm{~N}$ of HCL is poured on the plate and after 2-3 minutes, observations are made. The presence of a clear zone indicates a DNase positive result and its absence indicates a DNase negative result.

Antibiotic sensitivity testing: Antibiotic sensitivity was done on the isolates. The isolates were tested against penicillin $(10 \mu \mathrm{g})$.

Direct colony suspension method: The method was used in the preparation of the inoculum for seeding the antibiotic sensitivity agar plates. Eight to ten colonies (one at a time using sterile inoculating needle) from the nutrient agar plate of pure isolates were picked and inoculated into $5 \mathrm{ml}$ of sterile nutrient broth in a test tube. A sterile swab stick was then used to obtain the inoculum from the broth culture. To remove excess culture and enhance the formation of monolayer inoculum, the tip of the cotton wool applicator stick was pressed against the side of the test tube and then rotated. This was then streaked on the entire surface of a dried Mueller Hinton agar.

The disc diffusion method: With the aid of a sterile pair of forceps, a strip of the Gram-positive antibiotic disc (multidisc) was applied to the center of the seeded Mueller Hinton agar and gently pressed to the surface. The Mueller Hinton agar plates were then incubated at $37^{\circ} \mathrm{C}$ for $18-24$ hours. Zones of inhibition produced were measured and recorded. The zone of inhibition was measured and recorded. The zone of inhibition was interpreted as susceptibility or 
resistant based on the standard of the CLSI (Clinical and Laboratory Standard Institute).

\section{Results}

The result of the antibiotic sensitivity test showed that only $11.1 \%$ of the pure isolates were susceptible to penicillin $\mathrm{G}(10 \mu \mathrm{g})$. However, $88.9 \%$ of the isolates were resistant to penicillin G $(10 \mu \mathrm{g})$ (Table 1) (Table 2).

Table I Morphological and biological characteristic of the isolates

\begin{tabular}{|c|c|c|c|c|c|c|}
\hline Strain code & Gram reaction & Shape & Growth on MSA & Catalase & Coagulase & DNase \\
\hline UNS 6 & $+\mathrm{ve}$ & Cocci & + ve & + ve & $+\mathrm{ve}$ & $+\mathrm{ve}$ \\
\hline UNS 9 & + ve & Cocci & + ve & $+\mathrm{ve}$ & + ve & + ve \\
\hline UNS I4 & $+\mathrm{ve}$ & Cocci & $+\mathrm{ve}$ & + ve & $+\mathrm{ve}$ & $+\mathrm{ve}$ \\
\hline UNS I7 & $+\mathrm{ve}$ & Cocci & + ve & + ve & + ve & + ve \\
\hline UNS 4I & $+v e$ & Cocci & +ve & + ve & + ve & + ve \\
\hline UNS 50 & $+v e$ & Cocci & $+v e$ & $+v e$ & $+\mathrm{ve}$ & $+\mathrm{ve}$ \\
\hline UNS 89 & +ve & Cocci & +ve & +ve & +ve & +ve \\
\hline UNS I03 & $+v e$ & Cocci & $+\mathrm{ve}$ & $+\mathrm{ve}$ & $+\mathrm{ve}$ & $+v e$ \\
\hline UNS 105 & $+\mathrm{ve}$ & Cocci & +ve & $+v e$ & + ve & + ve \\
\hline
\end{tabular}

Table 2 Antibiotic susceptibility of the pure isolates

\begin{tabular}{|c|c|c|}
\hline $\mathbf{S} / \mathbf{N}$ & Sample code & Penicillin G ( $10 \mu g)$ \\
\hline I & UNS 6 & $\mathrm{R}$ \\
\hline 2 & UNS 9 & $\mathrm{R}$ \\
\hline 3 & UNS I4 & $\mathrm{R}$ \\
\hline 4 & UNS 17 & $\mathrm{R}$ \\
\hline 5 & UNS 4I & $\mathrm{R}$ \\
\hline 6 & UNS 50 & $\mathrm{R}$ \\
\hline 7 & UNS89 & $\mathrm{R}$ \\
\hline 8 & UNS 103 & $\mathrm{R}$ \\
\hline 9 & UNS 105 & $S$ \\
\hline $\mathbf{S} / \mathbf{N}$ & Sample code & Penicillin G ( $10 \mu g)$ \\
\hline I & UNS 6 & 13 \\
\hline 2 & UNS 9 & 11 \\
\hline 3 & UNS 14 & $|2|$ \\
\hline 4 & UNS 17 & 14 \\
\hline 5 & UNS 4I & 13 \\
\hline 6 & UNS 50 & 14 \\
\hline 7 & UNS 89 & 15 \\
\hline 8 & UNS 103 & 15 \\
\hline 9 & UNS 105 & 40 \\
\hline
\end{tabular}

\section{Discussion and conclusion}

The human nose serves as a habitat for a diverse group of microorganisms including $S$. aureus infection. It has been established that it is the major risk factor for the development of infection in certain groups of people. ${ }^{14}$ This clearly indicates that $88.9 \%$ of
Key: R, Resistant; S, Sensitive; I, Intermediate

Standard

S I $\quad$ R

$\geq 29-\quad \leq 28$

(Table 2 continuation)
Staphylococcus aureus in female students of O.A.U campus were resistant to penicillin. Staphylococcus aureus is a common bacterium. Most of us carry it in our noses or on our skin. But in the 1950's, less than 10 years after penicillin was made, Staphylococcus aureus had become so resistant to penicillin that healthy people going to hospitals got sick and died. Most strains of Staphylococcus aureus are now resistant to penicillin because, Staphylococcus aureus can make a substance called $\beta$-lactamase (pronounced beta- lactamase), that degrades penicillin, destroying its antimicrobial activity.

However, the reason for these could be lack of proper hygiene, delay in treatment and accessibility to drug without prescription. This is due to over population, lack of policies guiding drug use and management, lack of education, also high rates of multiple resistance is a direct consequence of hospital and community use of antibiotics and its concurrent selection of resistance strains where patients treat themselves with the wrong antibiotic or wrong dose and some patients could come to the hospital already harboring resistant strains. In order to prevent Staphylococcal related infection, people must should practice good and proper hygiene, have access to education, there should be policies guiding the use of drug and management etc. and this will also have a great impact in the future.

Therefore, the ability to control Staphylococcal infections in the future will depend on several factors e.g. development of new therapeutic agents, optimization of infection control measures and the introduction of new medical devices with a reduced risk of infection.

\section{Appendix}

$\begin{array}{ll}\text { Domain: } & \text { Bacteria } \\ \text { Kingdom: } & \text { Eubacteria } \\ \text { Phylum: } & \text { Fimicutes } \\ \text { Class: } & \text { Bacilli } \\ \text { Order: } & \text { Bacillales }\end{array}$




$\begin{array}{ll}\text { Family: } & \text { Staphylococcaceae } \\ \text { Genus: } & \text { Staphylococcus } \\ \text { Species: } & \text { Staphylococcus aureus }\end{array}$

\section{Acknowledgments}

None.

\section{Conflicts of interest}

Authors declare that there is no conflicts of interest.

\section{References}

1. Todar's K. Todar's online textbook of bacteriology: the genus Bacillus. Madison: University of Wisconsin; 2005.

2. Prescott L, Harley J, Klein D. Microbiology. 6th ed. Mc Graw-Hill Publishers; 2005.

3. Science Magazine; 2004.

4. Tolan R. Staphylococcus aureus infection. eMedicine Medscape; 2006.

5. Ryan KJ, Ray CG. Sherris Medical Microbiology. 4th ed. Mc GrawHill; 2004.

6. Jawetz E, Levinson W. Medical Microbiology \& Immunology: Examination and Board Review. University of Michigan; 1992. 472 p.
7. Katzung BG. Basic \& clinical pharmacology. New York: Lange Medical Books/McGraw Hill; 2004.

8. Neu HC. Penicillins-new insights into their mechanisms of activity and clinical use. Bull N Y Acad Med. 1982;58(8):681-695.

9. Monica C. Distinct laboratory practice in Tropical countries. Part 2. Canbridge University Press; 2000. 157-158 p.

10. Jones TA, Zou JY, Cowan SW, et al. Improved methods for building protein models in electron density maps and the location of errors in these models. Acta Crystallogr A. 1991;47(Pt 2):110-119.

11. Todar K. Bacteria resistance to antibiotics. Todar's online textbook of bacteriology; 2002.

12. Whitt A, Dixie D, Salyers B, et al. Bacterial pathogenesis: A Molecular Approach. 2nd ed. USA: ASM Press; 2002.

13. Raygada JL, Levine DP. Methicillin-Resistant Staphylococcus aureus: A Growing Risk in the Hospital and in the Community. Am Health Drug Benefits. 2009;2(2):86-95.

14. Von Eiff C, Becker K, Machka K, et al. Nasal carriage as a source of Staphylococcus aureus bacteremia. N Engl J Med. 2001;344(1):11-16.

15. Kluytmans J, van Belkum A, Verbrugh H. Nasal carriage of Staphylococcus aureus: epidemiology. Clin Microbiol Rev. 1997;10(3):505-520. 